\title{
Complement in Renal Disease as a Potential Contributor to Arterial Hypertension
}

\author{
Lisa-Maren Fischer $^{a} \quad$ Laura A. Fichte $^{a} \quad$ Maike Büttner-Herold ${ }^{a} \quad$ Fulvia Ferrazzi $^{a}$ \\ Kerstin Amann ${ }^{a}$ Kerstin Benz ${ }^{a, b} \quad$ Christoph Daniel $^{a}$ \\ aDepartment of Nephropathology, Institute of Pathology, Friedrich-Alexander-Universität Erlangen-Nürnberg, \\ Erlangen, Germany; ${ }^{b}$ Department of Pediatrics, Friedrich-Alexander-Universität Erlangen-Nürnberg, Erlangen, \\ Germany
}

\section{Keywords \\ Kidney $\cdot$ Complement deposition $\cdot$ Hypertension $\cdot$ Renal disease}

\begin{abstract}
Objective: Complement deposition is prevalent in kidney biopsies of patients with arterial hypertension and hypertensive nephropathy, but an association of hypertension and complement deposition or involvement of complement in the pathogenesis of hypertensive nephropathy has not been shown to date. Methods: In this study, we analyzed complement $\mathrm{C} 1 \mathrm{q}$ and $\mathrm{C} 3 \mathrm{c}$ deposition in a rat model of overload and hypertension by subtotal nephrectomy (SNX) and in archival human renal biopsies from 217 patients with known hypertension and 91 control patients with no history of hypertension using semiquantitative scoring of $\mathrm{C} 1 \mathrm{q}$ and $\mathrm{C} 3 \mathrm{C}$ immunohistochemistry and correlation with parameters of renal function. To address whether complement was only passively deposited or actively expressed by renal cells, C1q and C3 mRNA expression were additionally analyzed. Results: Glomerular C1q and C3c complement deposition were significantly higher in kidneys of hypertensive SNX rats and hypertensive compared to nonhypertensive patients. Mean arterial blood pressure (BP) in SNX rats correlated well with the
\end{abstract}

amount of glomerular C1q and C3c deposition and with left ventricular weight, as an indirect parameter of high BP. Quantitative mRNA analysis showed that C3 was not only deposited but also actively produced by glomerular cells of hypertensive SNX rats and in human renal biopsies. Of note, in patients CKD-stage correlated significantly with the intensity of glomerular $\mathrm{C} 3 \mathrm{c}$ staining, but not with that of $\mathrm{C} 1 \mathrm{q}$. Conclusion: Renal complement deposition correlated with experimental hypertension as well as the presence of hypertension in a variety of renal diseases. To answer the question, if and how exactly renal complement is causative for the pathogenesis of arterial hypertension in men, further studies are needed.

\footnotetext{
(C) 2021 The Author(s).

Published by S. Karger AG, Basel
}

\section{Introduction}

The complement system and its regulatory proteins are components of the innate immune system consisting of $>20$ soluble factors, playing an important role in (i) opsonization, (ii) stimulation of different inflammatory

Kerstin Benz and Christoph Daniel: equal contribution. karger@karger.com www.karger.com/kbr

Karger ${ }^{\prime}$ -

BOPEN ACCESS
(C) 2021 The Author(s)

Published by S. Karger AG, Basel

This is an Open Access article licensed under the Creative Common Attribution-NonCommercial-4.0 International License (CC BY-NC) (http://www.karger.com/Services/OpenAccessLicense), applicable to the online version of the article only. Usage and distribution for commercial purposes requires written permission.
Correspondence to:

Christoph Daniel, christoph.daniel@uk-erlangen.de 
pathways, and (iii) osmolytic lysis of pathogens and damaged cells in numerous diseases, especially inflammatory kidney disease [1]. The complement system consists of 3 pathways: (i) the classical pathway, activated by any structure that is recognized by C1q [2], (ii) the lectin pathway, activated when saccharide patterns are recognized by pattern recognition complexes [3], and (iii) the alternate pathway, activated through spontaneous hydrolysis of C3 [4].

The past 2 decades intensified our knowledge of the complement system and its role in the pathogenesis of different systemic and renal diseases like postinfectious glomerulonephritis (GN), IgA nephropathy (IgAN), lupus nephritis (LN), membranoproliferative $\mathrm{GN}$, and $\mathrm{C} 3$ glomerulopathies $[1,5]$. An important role of the complement system and its dysfunction is also known in the pathogenesis of thrombotic microangiopathies, including the atypical hemolytic uremic syndrome [6], IgA vasculitis (former Henoch-Schönlein purpura) [7], and malignant hypertension [8]. While pathomechanisms of thrombotic microangiopathy are already known [6, 7], our knowledge of the significance of the complement system in relation to hypertension, is still relatively vague. The majority of patients with CKD present with elevated blood pressure (BP) [9], but the exact pathogenesis of hypertension is unknown. Several studies are available describing an association of plasma $\mathrm{C} 3$ and hypertension $[10,11]$, while another study could not confirm these observations [12]. In the rat DOCAsalt hypertension model, glomerular $\mathrm{C} 3$ deposition was increased in DOCA-salt rats and decreased by antihypertensive therapy, that is, spironolactone and triple therapy with hydrochlorothiazide, reserpine, and hydralazine [13]. Recent studies using preclinical models of hypertension suggested a role of complement in the pathogenesis of hypertension [14] mediated by different immune cells like macrophages and regulatory Tcells (Tregs) [15-17] and activation of the renin-angiotensin system leading to phenotypic changes in vascular smooth muscle cells [18]. The detailed role of renal complement deposition in the development of elevated $\mathrm{BP}$ is still unclear. Therefore, in this study, we investigated $\mathrm{C} 1 \mathrm{q}$, as a cleavage product of the classical, and $\mathrm{C} 3 \mathrm{c}$ as a common marker of all 3 complement activation pathways in renal biopsies from hypertensive and normotensive patients as well as in an established rat model of overload and subsequent hypertension by subtotal nephrectomy (SNX).

Renal Complement Deposition in

Hypertension

\section{Methods}

\section{Animal Model}

For investigation of renal complement deposition, renal injury, and cardiac changes in hypertensive animals, we used the rat model, which is characterized by glomerular hyperfiltration and overload due to nephron reduction, focal segmental and global glomerulosclerosis, tubulointerstitial scarring and progressive CKD with significantly increased BP. Rats were maintained in a specific pathogen-free facility in a temperature- and light-controlled environment and had ad libitum access to standard chow (sniff Spezialdiäten GmbH, Soest, Germany) and water. The rats were kept on classical aspen wood bedding (sniff Spezialdiäten $\mathrm{GmbH}$, Soest, Germany) in type IV makrolon cages (Tecniplast Deutschland GmbH, Hohenpeißenberg, Germany) with a maximum population of 3. For model induction, 12 male Dark Agouti (DA/HanRj, Janvier, Le Genest-Saint-Isle, France) rats with an age of 12-14 weeks were SNX in a 2 step protocol starting with uninephrectomy followed by weight standardized resection of the upper and lower poles from the remaining kidney 1 week later. For this purpose, the animals were administered Buprenovet (Bayer, Leverkusen, Germany) in a dose of $0.05 \mathrm{mg} / \mathrm{kg}$ body weight s.c. before surgery. After anesthesia with isoflurane, the right flank was shaved, disinfected with KODAN tincture forte (Schülke \& Mayr GmbH, Norderstedt, Germany), the flank was opened with a $1.5 \mathrm{~cm}$ incision and the kidney carefully lifted out and fixed with 2 sterile swabs followed by ligation of the renal blood supply and ureter and removal of the kidney. The abdominal incision was closed with an absorbable suture and a continuous seam as well as the skin closed with single button seams. One week later, the left kidney was lifted out exactly as described for the right kidney. Then the renal artery was clamped with an atraumatic clip and upper and lower pole of the kidney was resected with a scalpel and the cut surface was closed with a collagen sponge (Resorba medical GmbH, Nürnberg, Germany). Finally, the remaining kidney was returned to its original position and the abdominal incision was closed as described above. Postoperative activity, fluid intake, weight, coat texture, and behavior of the animals were assessed but showed no signs of postoperative pain. However, analgesia was maintained at least over 3 days after surgery. Controls received a sham surgery (sham, $n=11$ ). At the end of the experiment, animals were housed for $24 \mathrm{~h}$ in metabolic cages to collect urine for measurement of proteinuria using a Bio-Rad Protein Assay (Bio-Rad Laboratories GmbH, Munich, Germany). Adsorption was measured in PBS-diluted urine samples at $595 \mathrm{~nm}$ in a microplate reader, and proteinuria was calculated using a standard curve from bovine serum albumin standards ranging from 0.15 to $2 \mathrm{mg}$ (Thermo Scientific, Rockford, IL, USA). After 12 weeks, rats were euthanized after intra-arterial BP measurement for $30 \mathrm{~min}$ via insertion of a catheter into A. carotis using $\mathrm{AD}$ instruments power lab system (AdInstruments Ltd., Oxford, UK). Finally, the kidneys and hearts were harvested and split into 2 parts, one was snap frozen for RNA analysis and the second part was processed for immunohistochemistry. Blood was collected for detection of serum creatinine using an autoanalyzer (Beckman Instruments, Brea, CA, USA).

\section{Quantitative mRNA Analysis}

Total RNA from rat kidneys was isolated using RNeasy Mini columns (Qiagen, Hilden, Germany). Reverse transcription reactions and real-time PCR were performed using Power SYBR Green on a 7500 Fast Real-Time PCR system (both Applied Biosystems, Weiterstadt, Germany) according to the manufacturer's instructions. Real-time PCR data were analyzed using the SDS v1.3 soft- 
Table 1. Patient characteristics

\begin{tabular}{llllll}
\hline No. & Diagnosis & $\begin{array}{l}\text { Hypertensive } \\
\text { patients, } N / n\end{array}$ & $\begin{array}{l}\text { Percentage of } \\
\text { hypertension, } \%\end{array}$ & $\begin{array}{l}\text { Sex }(\mathrm{m} / \mathrm{w}), \\
n / n\end{array}$ & $\begin{array}{l}\text { Age, years } \\
(\mathrm{mean} \pm \mathrm{SD})\end{array}$ \\
\hline $\begin{array}{l}\text { Controls } \\
1\end{array}$ & & & & \\
\hline Nephropathies & & & & & \\
2 & Zero-biopsy & $46 / 0$ & & $49 / 16$ & $49.7 \pm 17.2$ \\
3 & IgAN & $65 / 58$ & 89.2 & $12 / 6$ & $55.8 \pm 18.2$ \\
4 & MGN & $18 / 11$ & 61.1 & $5 / 5$ & $39.2 \pm 15.2$ \\
5 & LN & $10 / 8$ & 80.0 & $14 / 14$ & $60.8 \pm 12.8$ \\
6 & DN & $28 / 26$ & 92.9 & $10 / 7$ & $52.5 \pm 21.1$ \\
7 & FSGS & $17 / 15$ & 88.2 & $77 / 47$ & $53.5 \pm 18.5$ \\
\hline & Others* & $124 / 99$ & 79.8 & $\mathbf{1 9 0 / 1 1 8}$ & $\mathbf{5 3 . 5} \pm \mathbf{1 7 . 9}$ \\
\hline
\end{tabular}

TMA, thrombotic microangiopathy; MGN, membranous GN; LN, lupus nephritis; FSGS, focal segmental glomerulosclerosis; IgAN, IgA nephropathy; DN, diabetic NP. * Others: membranoproliferative GN, postinfectious GN, C3 GN, ANCA-associated vasculitis, IgA vasculitis (HSPN), hypertensive nephropathy (NP), TMA, transplant NP, amyloidosis, end-stage renal disease (of unknown origin), Minimal change disease, M. Alport, thin basement disease, acute tubular injury, interstitial nephritis, cholesterol embolism, oxalate NP, light chain cast NP.

ware (Applied Biosystems), and relative expression of target gene mRNA levels was calculated using the comparative delta Ct method [19]. Normalization was conducted against the endogenous $18 \mathrm{~S}$ rRNA levels applied to the resulting relative fold changes. The following primers were used: $18 \mathrm{~s}$ (fw $5^{\prime}$ TTGATTAAGTCCCTGCCCTTTGT3'; rev 5'CGATCCGAGGGCCTCACTA3'); C3 (fw 5' TGCGGCTGGAGAGTGAAG3' ${ }^{\prime}$; rev $5^{\prime}$ TTACTGGCTGGAATCTTGATGG3') [20]; and C1qB (fw 5'TCATAGAACACGAGGATTCCATACA3'; rev 5'GACCCAGTACAGCTGCTTTGG3') [20].

\section{Immunohistochemistry}

For immunohistochemistry kidney biopsies or SNX and sham rat kidney, specimens were fixed in formalin, embedded in paraffin and cut into sections of $1 \mu \mathrm{m}$ and stained in a Ventana Benchmark stainer (Roche Diagnostics Deutschland GmbH, Mannheim, Germany) using the following protocol: antigen retrieval was done using pronase $\mathrm{E}$ digestion for $40 \mathrm{~min}$ at $37^{\circ} \mathrm{C}$. After blocking with AvidinBiotin Block (Vector Laboratories, Burlingame, CA, USA), normal goat serum and blotto (1:5) sections were incubated for $1 \mathrm{~h}$ at $37^{\circ} \mathrm{C}$ using the following antibodies diluted 1\% BSA in $50 \mathrm{~mm}$ Tris $\mathrm{pH}$ 7.4: $\mathrm{C} 1 \mathrm{q}$, a rabbit polyclonal antibody against human $\mathrm{C} 1 \mathrm{q}$ (A0126; DAKO Deutschland, Hamburg, Germany) and; C3c, a rabbit polyclonal antibody against human C3c (A0062; DAKO Deutschland). After washing with $50 \mathrm{~mm}$ Tris $\mathrm{pH} 7.4$, bound primary antibodies were detected using the "ultraView Universal DAB detection Kit" (Roche Diagnostics Deutschland GmbH, Mannheim, Germany). Negative controls included either deletion or substitution of the primary antibody with equivalent concentrations of an irrelevant murine monoclonal antibody or preimmune rabbit IgG.

\section{Human Renal Tissue Specimens}

In a first step, an unselected cohort of 308 consecutive formalinfixed paraffin-embedded archival kidney biopsies (Department of $\mathrm{Ne}$ - phropathology, Friedrich-Alexander-Universität Erlangen-Nürnberg [FAU]) was analyzed for renal complement deposition using immunohistochemistry and comparing cases with presence or absence of arterial hypertension according to the clinical files, irrespective of the primary renal disease. Only biopsies from hypertensive and nonhypertensive patients with clinically well-documented BP were included. In addition, 46 zero-biopsies from transplant kidney allografts donated from nonhypertensive living donors served as controls.

In a second step, we focused on the comparison of selected common groups of renal diseases, namely controls (Zero-biopsies; $n=46)$, IgAN $(n=65)$, membranous GN (MGN, $n=18), \mathrm{LN}(n=$ 10 ), focal segmental glomerulosclerosis (FSGS, $n=17$ ) and diabetic nephropathy ( $\mathrm{DN}, n=28$ ) resulting in a representative subgroup of 184 biopsies. In this subgroup, the relation of arterial hypertension and complement deposition in the context of different common nephropathies was analyzed in more detail. Patients' basic characteristics are described in Table 1.

\section{Histopathological Evaluation}

Renal biopsy diagnostic was performed by 3 very experienced, specialized nephropathologists during routine work-up with consensus discussion of all ambiguous cases (including M.B.H. and K.A.). Assessment of detailed morphology of human renal biopsies was done by one very experienced scientist (CD) using well-established semiquantitative glomerulosclerosis score, tubular injury score, and vascular injury score (VSI) on PAS-stained paraffin sections as described previously [21]. Interstitial fibrosis/tubular atrophy (IF/TA) was graded according to BANFF classification for renal transplant biopsies [22].

\section{Evaluation of Retrospective Clinical Data}

Clinical parameters that were available at the time-point of renal biopsy were collected. The following parameters were includ- 


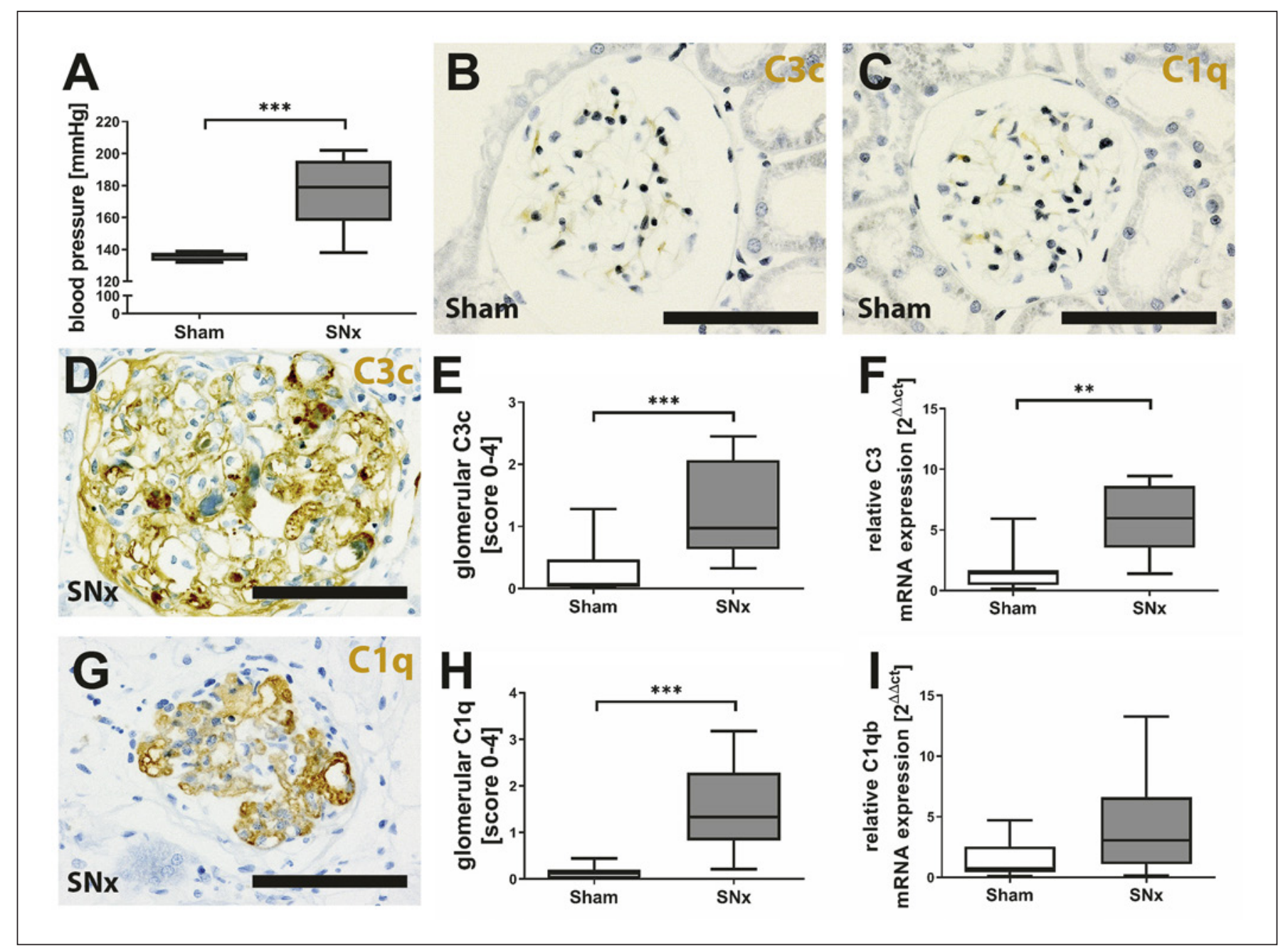

Fig. 1. Complement was markedly deposited in the kidneys of SNX rats. Mean arterial BP was significantly higher in SNX rats than sham-operated controls (A). Representative pictures of glomerular $\mathrm{C} 3 \mathrm{C}(\mathbf{B}, \mathbf{D})$ and $\mathrm{Clq}(\mathbf{C}, \mathbf{G})$ in sham-op control $(\mathbf{B}, \mathbf{C})$ and SNX rats $(\mathbf{D}, \mathbf{G})$ are shown (brown staining). Glomerular C3c deposition (E) and C3 mRNA expression (F) as well as glomerular C1q deposition
(H) and C1qb mRNA expression (I) were analyzed in sham-operated control and SNX rats. Significant differences are marked by asterisks: ${ }^{* *} p<0.01$ and ${ }^{* * *} p<0.001$ indicate significant differences between sham-operated rats with normal BP and hypertensive SNX rats. Scale bars represent $50 \mu \mathrm{m}$. SNX, subtotal nephrectomy; BP, blood pressure. ed for correlation analysis with C1q-and C3c-scores, complement localization, and injury scores using SPSS software: patient age, presence or absence of hypertension and diabetes, proteinuria, serum creatinine, serum urea, serum cholesterol, serum protein, and serum C3 and C4 complement factors. Unfortunately, information on the duration of renal disease and current medication was not consistently available and could therefore not be systematically analyzed. In renal patients, BP is measured regularly and particularly before performing a kidney biopsy. BP measurements and grading was done according to the KDIGO guidelines for renal patients [23]. Proteinuria was measured by analysis of a $24 \mathrm{~h}$ urine collection and significant proteinuria was defined as urinary protein excretion $>300 \mathrm{mg} /$ day. In $2 / 3$ of all cases, proteinuria was given as $\mathrm{g} / 24 \mathrm{~h}$ urine. Only some patients had proteinuria docu-

Renal Complement Deposition in Hypertension mented by spot urine measurements, and for very few patients, there was no data on proteinuria available. Since $24 \mathrm{~h}$ and spot proteinuria values can be hardly compared, we only included patients with $24 \mathrm{~h}$ urine collection for correlation of proteinuria with complement deposition. In order to further standardize our findings, we also subdivided the patients according to different CKD classes using the CKD-EPI formula for calculation of GFR [24] and the CKD classes according to the KDIGO 2012 classification [25].

\section{Semiquantitative Evaluation of Complement Deposition in} Renal Biopsies

Complement staining by immunohistochemistry in renal biopsies was graded separately in glomeruli and the tubulointer- 


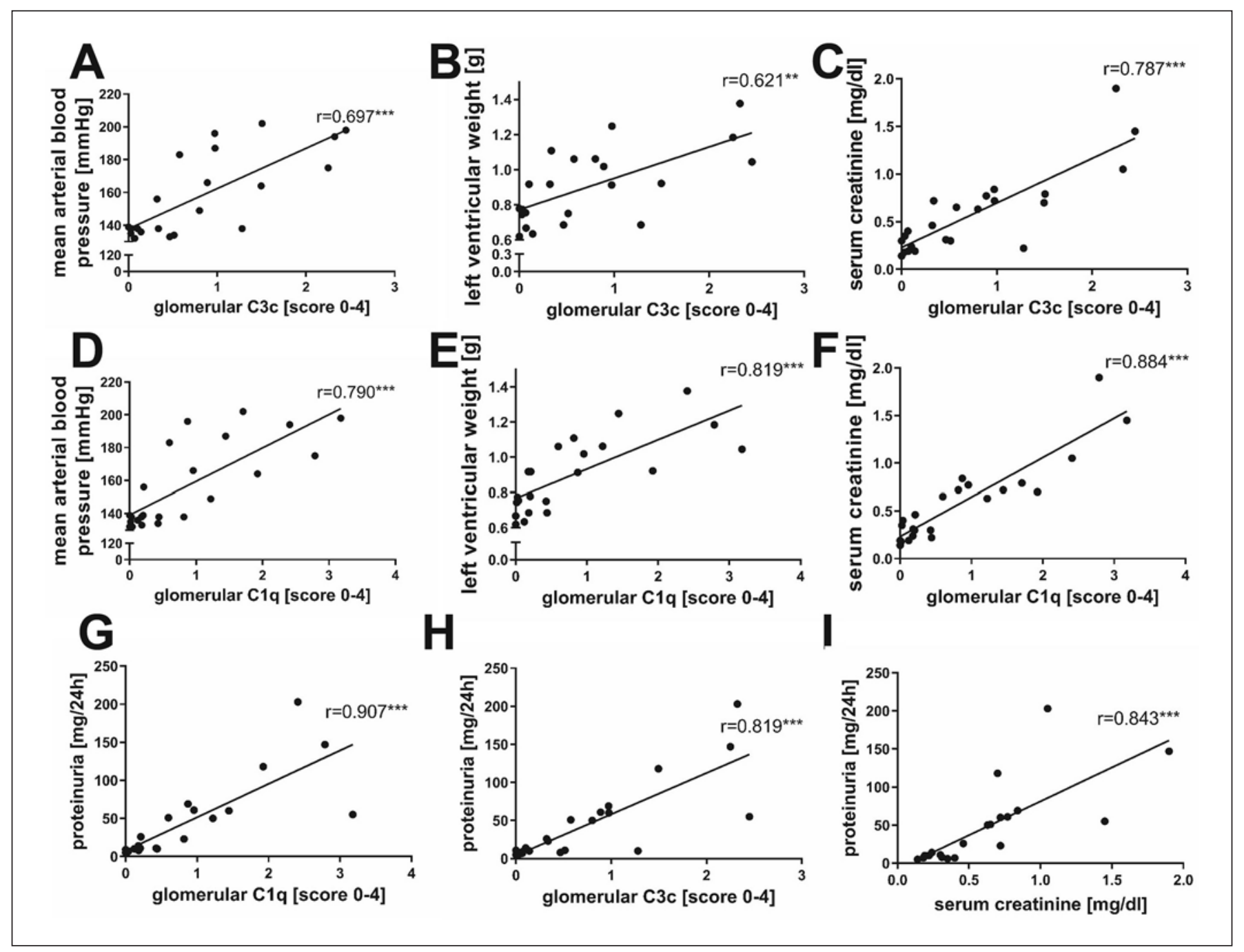

Fig. 2. Complement was markedly deposited in the kidneys of SNX rats and correlated with cardiac changes and renal function. Glomerular C3c correlated with mean arterial BP (A), LV weight (B), serum creatinine $(\mathbf{C})$, and proteinuria $(\mathbf{H})$. Similarly, glomerular C1q correlated with mean arterial BP (D), LV weight (E), serum creatinine $(\mathbf{F})$, and proteinuria (G). Serum creatinine correlated well with proteinuria (I). Significant correlations, as assessed by spearman's test, are marked by asterisks: ${ }^{* *} p<0.01$ and ${ }^{* * *} p<$ 0.001. SNX, subtotal nephrectomy; BP, blood pressure.

\section{Statistical Analyses}

After testing for normal distribution of values using Kolmogorov-Smirnov test, data were analyzed using Mann-Whitney test for comparison of 2 groups and Kruskal-Wallis test with Dunn's Multiple Comparison test as post hoc test for comparison of multiple groups. In all tests, $p<0.05$ was accepted as statistically significant. Spearman's test was used to test correlation of complement deposition with renal injury scores and clinical data. Statistical analyses were performed using SPSS for Windows software (version 19.0 SPSS, IBM, Munich, Germany) or GraphPad Prism 8 for Windows software (version 8.3, GraphPad Software Inc., San Diego, CA, USA).
C1q and C3 mRNA in Human Renal Biopsies

Data for $\mathrm{C} 1 \mathrm{q}$ and $\mathrm{C} 3$ in glomeruli and tubulointerstitium of human renal biopsies with different diseases were evaluated from Nephroseq database using the Ju CKD dataset [26].
Fischer/Fichte/Büttner-Herold/Ferrazzi/ Amann/Benz/Daniel 


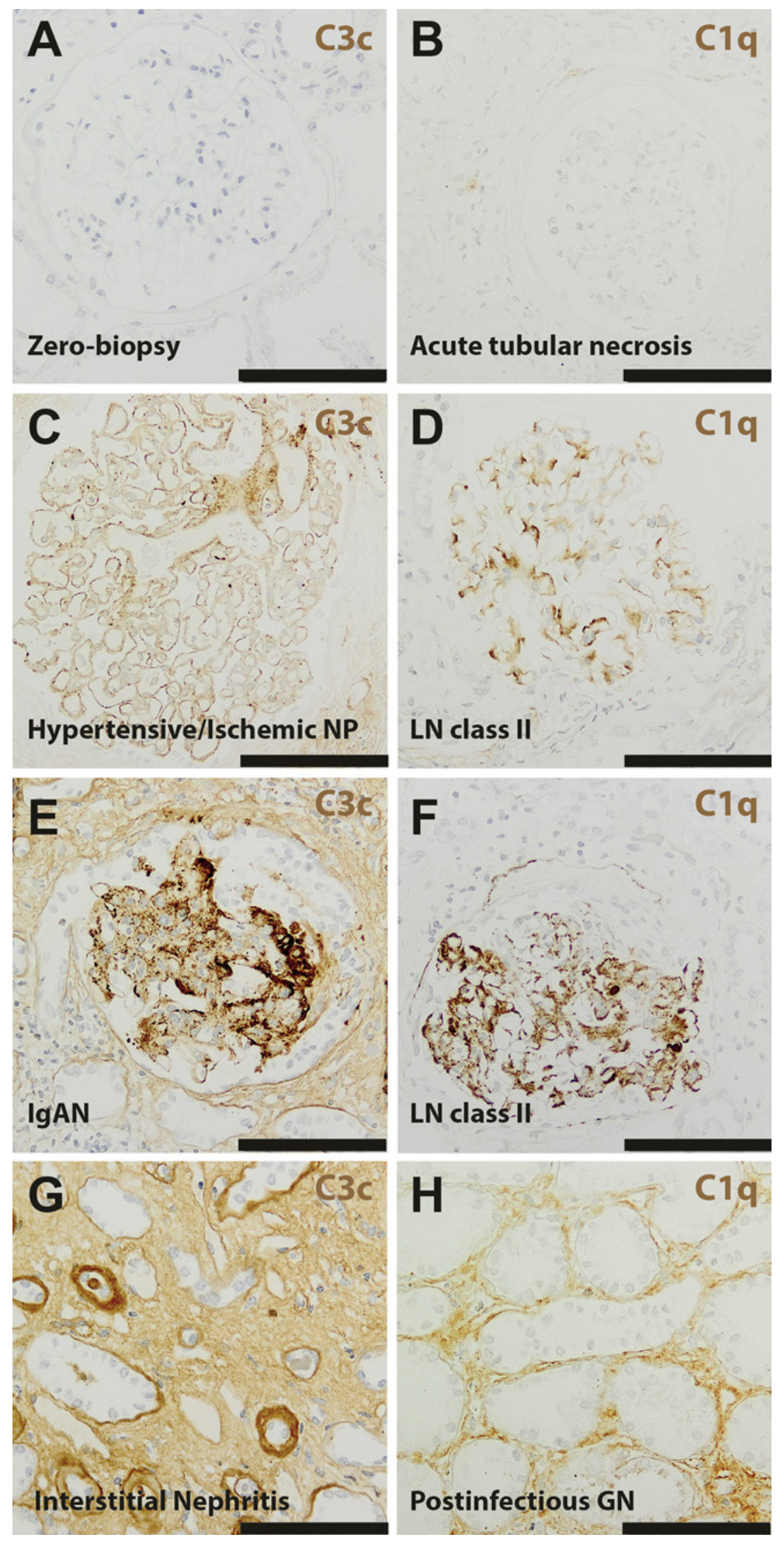

Fig. 3. $\mathrm{C} 3 \mathrm{c}$ and $\mathrm{C} 1 \mathrm{q}$ deposition in renal biopsies. Human renal biopsies were stained for C3c (A, C, E, G) and C1q (B, D, F, H) using immunohistochemistry (brown staining). Representative pictures of glomerular and tubulointerstitial deposition of $\mathrm{C} 3 \mathrm{c}$ and $\mathrm{C} 1 \mathrm{q}$ show examples of different scores of complement deposition. No staining for $\mathrm{C} 3 \mathrm{c}$ in zero-biopsies $(\mathbf{A})$ and $\mathrm{C} 1 \mathrm{q}$ in acute tubular necrosis (B). Examples for glomerular C3c deposition in hypertensive/ischemic $\mathrm{NP}$, score 1 (C) and C1q deposition in class II $\mathrm{LN}$, score 2 (D). More prominent $\mathrm{C} 3 \mathrm{c}$ deposition in IgAN, score 4 (E) and C1q deposition, score 4, in another case of class II LN (F). Deposition in the tubulointerstitial compartment of $\mathrm{C} 3 \mathrm{c}$ in interstitial nephritis, score $4(\mathbf{G})$ and $\mathrm{Clq}$ deposition, score $4(\mathbf{H})$ in a case of postinfectious GN. Scale bars represent $100 \mu \mathrm{m}$. LN, lupus nephritis; GN, glomerulonephritis. 


\section{Results}

Renal Complement C3c and C1q Deposition Is Present in a Hypertensive Rat Model of Stable CKD and

Correlates with Renal and Cardiac Changes

Complement deposition was investigated in a hypertensive rat model of CKD, that is, subtotally nephrectomized (SNX) Dark Agouti rats, in which mean arterial BP 12 weeks after model induction was significantly $(p<0.001)$ increased $(175.7 \pm 21.0 \mathrm{~mm} \mathrm{Hg})$ compared to sham-operated controls $(135.8 \pm 2.8 \mathrm{~mm} \mathrm{Hg})$ (Fig. 1A). Proteinuria was also significantly higher in SNX compared to sham-operated controls $(78.5 \pm 16.6$ vs. $8.9 \pm 0.8 \mathrm{mg} / 24 \mathrm{~h}$ ). This overload rat model of hypertension and progressive renal insufficiency seemed suitable to examine hypertension-associated changes in renal complement deposition and expression. Glomerular $\mathrm{C} 3 \mathrm{c}$ and $\mathrm{C} 1 \mathrm{q}$ deposition were absent in normotensive, sham-operated control rats (Fig. 1B, C), and significantly increased in hypertensive SNX rats (Fig. 1D, E, G, H). In SNX rats, complement factor C3c was not only deposited in renal tissue but also significantly upregulated on mRNA level (Fig. 1F). Renal C1q mRNA expression showed a tendency to higher levels in SNX rats than controls, but this difference did not reach significance (Fig. 1I). Interestingly, in rats both glomerular $\mathrm{C} 3 \mathrm{c}$ and $\mathrm{C} 1 \mathrm{q}$ deposition highly correlated with mean arterial BP (Fig. 2A, D) and cardiac hypertrophy as assessed by LV weight (Fig. 2B, E). Furthermore, in the SNX model, renal complement deposition is correlated with kidney injury as well as renal function. Glomerular $\mathrm{C} 3 \mathrm{c}$ as well as $\mathrm{C} 1 \mathrm{q}$ deposition showed a strong correlation with serum creatinine (Fig. 2C, F) and proteinuria (Fig. 2G, H). Correlation of both serum creatinine and proteinuria with complement was not surprising since both parameters also showed strong correlation with each other in this rat model (Fig. 2I). Thus, our data in a rat model of hypertensive CKD due to kidney mass reduction and overload suggested an association of renal complement deposition and hypertension. Therefore, we aimed to confirm these findings in a human cohort of hypertensive

Fig. 4. Renal complement deposition and expression in different kidney diseases. Semiquantitative analysis of glomerular C3c (A) and C1q staining (B) in selected renal diseases. IgAN, MGN, LN, DN, FSGS and biopsies from transplant kidney allografts donated from nonhypertensive donors (0-biopsy) served as controls. Data from Nephroseq database showed glomerular C3c (C) and C1q (D) mRNA expression in different renal diseases and the respective control group, that is, HLD. In the tubulointerstitial compartment, versus normotensive patients, in whom a renal biopsy was performed for various reasons.

\section{Complement Deposition Is Independent of the Underlying Renal Disease}

Here, we used a 2 step approach: first, we included all patients with a reported BP value independent of the underlying kidney disease ( $n=308$, see Table 1 for details). Of note, 217 of 308 patients (70.5\%) were reported to have hypertension. Second, for further analysis, we focused on subgroups of most prevalent forms of GN, that is, IgAN, MGN, and LN, and 2 nonimmune complex-mediated renal diseases namely $\mathrm{DN}$ and focal segmental glomerulosclerosis (FSGS). 46 zero-biopsies from transplant kidney allografts donated from normotensive living donors served as a control group (see Table 1).

To investigate a potential relationship between hypertension and renal complement, deposition of $\mathrm{Clq}$ and $\mathrm{C} 3 \mathrm{c}$ in the glomerular and tubulointerstitial compartment was analyzed by semiquantitative scoring of immunohistological stainings. The amount and intensity of $\mathrm{C} 1 \mathrm{q}$ and $\mathrm{C} 3 \mathrm{c}$ staining varied greatly in the investigated biopsies (for details see Fig. 3). In the tubulointerstitial compartment, $\mathrm{C} 3 \mathrm{c}$ deposited along the tubular basement membranes and within the interstitial matrix (Fig. 3G), while $\mathrm{C} 1 \mathrm{q}$ staining was usually faint and restricted to the interstitium (Fig. $3 \mathrm{H}$ ).

Glomerular C3c deposition was increased in many renal diseases, including IgAN, MGN, LN, and FSGS, compared to normotensive zero-biopsies (Fig. 4A). Overall, glomerular $\mathrm{Clq}$ deposition was fainter than glomerular C3c. It was significantly increased in IgAN, MGN, LN, and DN, but not FSGS compared to zero-biopsy controls (Fig. 4B). Interestingly, complement $\mathrm{C} 3$ and $\mathrm{C} 1 \mathrm{q}$ were not only deposited in diseased kidneys, but local renal mRNA expression was also increased. Nephroseq data showed that both, $\mathrm{C} 3$ and $\mathrm{C} 1 \mathrm{q}$ mRNA expression, was significantly upregulated in IgAN, LN, DN, and FSGS compared to the control group, that is, healthy living donors (Fig. 4C, D). C1q mRNA expression was lower than C3 expression. Tubulointerstitial C3c deposition was also increased com-

primarily C3c (E) was detected in renal biopsies from patients with various diseases. Significant differences are marked by asterisks: ${ }^{*} p<0.05 ;{ }^{* *} p<0.01$ and ${ }^{* * *} p<0.001$ indicate significant differences versus zero-biopsies. GN, glomerulonephritis; MGN, membranous GN; LN, lupus nephritis; FSGS, focal segmental glomerulosclerosis; DN, diabetic nephropathy; HLD, healthy living donor; IgAN, IgA nephropathy.

(For figure see next page.) 
A

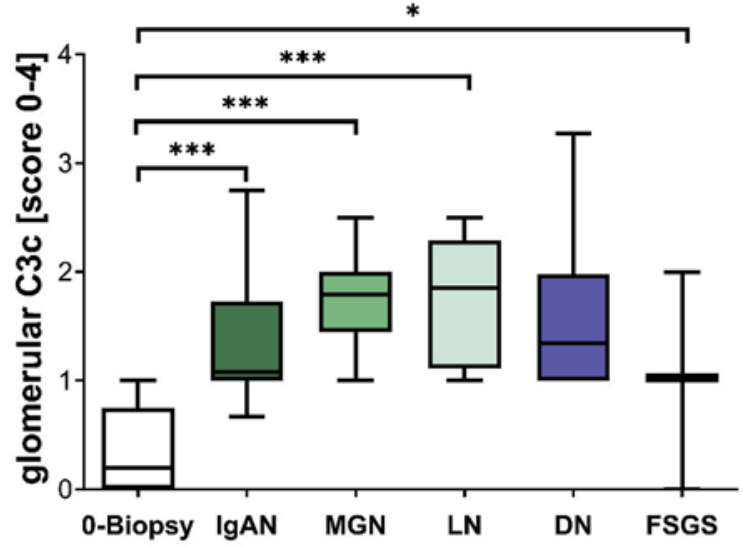

C

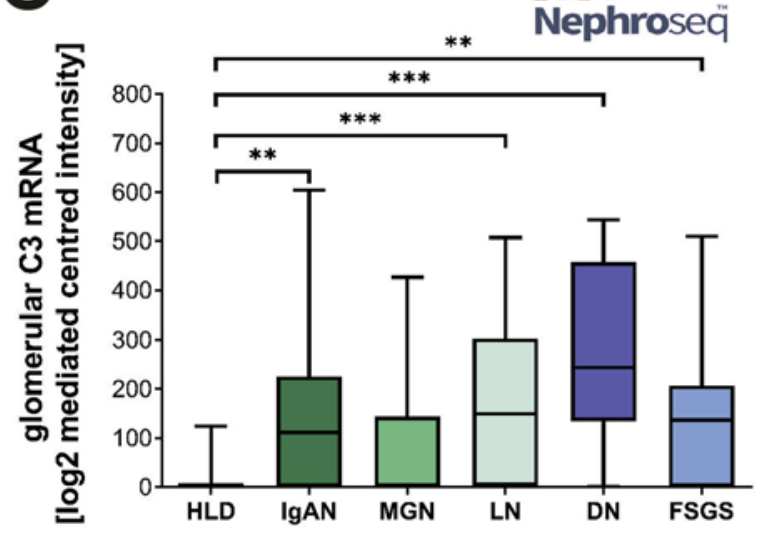

E

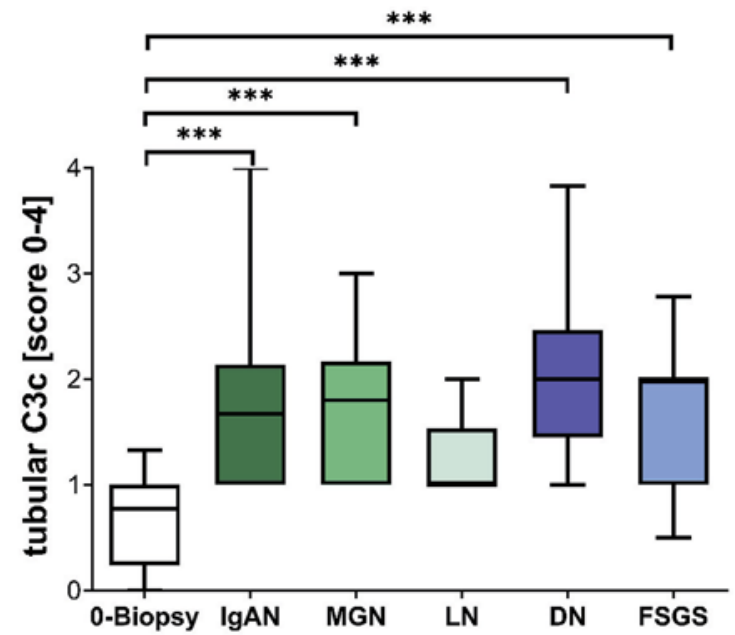

B

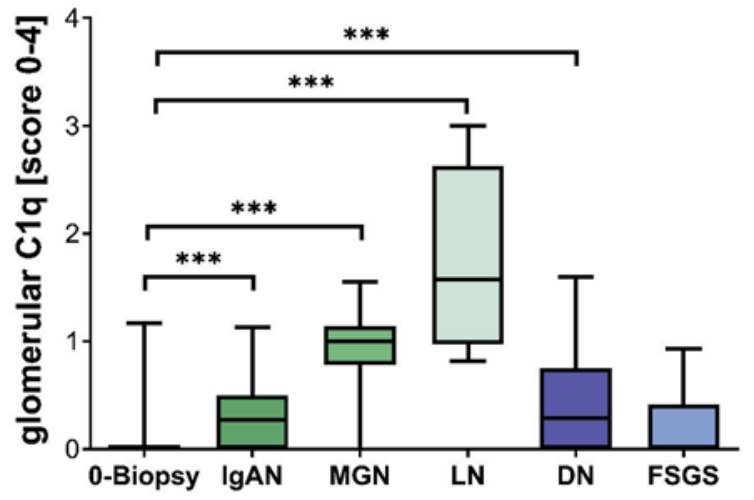

D

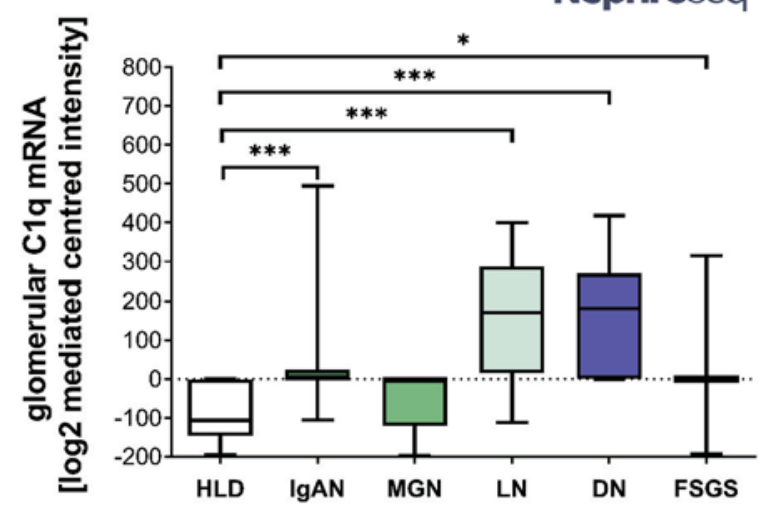




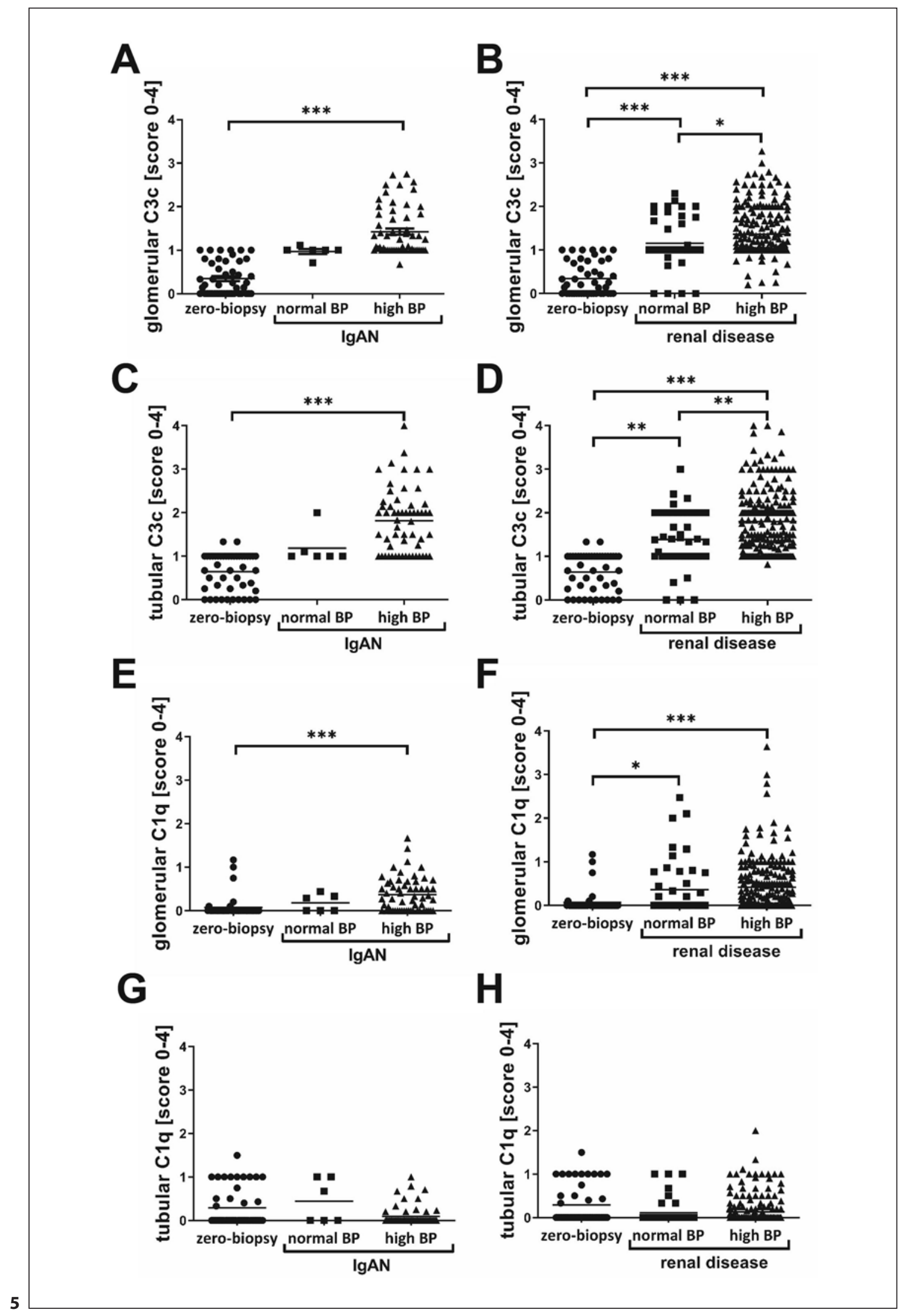

(For legend see next page.) 
pared to zero-biopsies in most renal diseases (Fig. 4E), while C1q was not significantly different from zero-biopsy controls (data not shown). In summary, marked renal complement deposition is a common finding in various kidney diseases. Next, we investigated the potential relevance of high $\mathrm{BP}$ on renal complement deposition.

\section{Renal C3c and C1q Deposition Are Significantly \\ Increased in Hypertensive Compared to Normotensive Patients}

To investigate whether presence of high $\mathrm{BP}$ in patients is associated with renal complement deposition, we analyzed biopsies from normotensive and hypertensive patients with various underlying kidney diseases and compared them with complement deposition in zero-biopsies from transplant kidney allografts donated from nonhypertensive donors (zero-biopsies). In IgAN, the most prevalent GN in our region [27], only biopsies from patients with hypertension had significantly higher glomerular (Fig. 5A) and tubulointerstitial C3c (Fig. 5C) as well as glomerular $\mathrm{C1}$ q deposition (Fig. 5E) compared to zerobiopsies. When we compared C3c (Fig. 5B, D) and C1q deposition (Fig. 5F) in renal biopsies of the total cohort, both normotensive and hypertensive samples showed significantly increased reactivity when compared to controls. Moreover, in biopsies of hypertensive patients glomerular and tubulointerstitial, C3c was significantly higher than normotensive patients (Fig. 5B, D). Since tubular $\mathrm{C} 1 \mathrm{q}$ deposition was low in all investigated biopsies, we did not observe any differences between groups neither in IgAN (Fig. 5G) nor in the analysis of all investigated cases (Fig. $5 \mathrm{H}$ ). Therefore, our data suggest that in particular $\mathrm{C} 3 \mathrm{c}$ deposition seems to be relevant in hypertensive renal diseases.

Fig. 5. Renal complement deposition in kidney biopsies from hypertensive and normotensive patients. $\mathrm{C} 3 \mathrm{c}$ deposition was analyzed in biopsies from patients with $\operatorname{IgAN}(\mathbf{A}, \mathbf{C})$ and all investigated biopsies with renal diseases $(\mathbf{B}, \mathbf{D})$ separated in patients with normal BP and high BP compared to biopsies from transplant kidney allografts donated from nonhypertensive donors (zero-biopsies) as controls in the glomerular (A, B) and tubulointerstitial

Fig. 6. Renal complement deposition in human biopsies correlated with renal damage and function. Renal damage as assessed by GSI (A), TSI (B), and VSI (C) was significantly increased in patients with high BP and GSI correlated with glomerular C3c deposition (D), TSI correlated with tubulointerstitial C3c (E), and VSI correlated with tubulointerstitial C3c (F). IF/TA (G) and serum creatinine $(\mathbf{H})$ were increased in hypertensive patients, while proteinuria was similar in both groups (I). Tubular C3c correlated with IF/TA
Renal Complement Deposition Is Associated with Kidney Injury and Function

The evaluation of histopathological changes, as assessed by glomerulosclerosis (Fig. 6A), tubulointerstitial injury score (Fig. 6B), and VSI (Fig. 6C), showed significant higher renal injury in biopsies from patients with documented hypertension than normotensive patients. The extent of glomerular C3c deposition correlated positively with glomerulosclerosis (Fig. 6D) and tubulointerstitial C3c with tubulointerstitial injury (Fig. 6E) and VSI (Fig. 6F), indicating that complement deposition is associated with chronic kidney injury. In addition, interstitial fibrosis/tubular atrophy (IF/TA) was significantly higher in renal biopsies of hypertensive patients (Fig. 6G) and correlated well with the extent of tubular $\mathrm{C} 3 \mathrm{c}$ deposition (Fig. 6J). Kidney function, as assessed by serum creatinine, was also significantly lower in hypertensive patients (Fig. 6H) and correlated with glomerular C3c deposition (Fig. 6K). No significant difference between normotensive and hypertensive patients was detected with regard to proteinuria (Fig. 6I). However, there was a weak correlation between proteinuria and glomerular C3c $(r=0.238 ; p=0.008$; Fig. $6 \mathrm{~L})$. These results confirmed an association of kidney injury, function, complement deposition, and hypertension.

Finally, we investigated the extent to which complement deposition was dependent on CKD stage. As expected, the mean age of patients increased with higher CKD stage (Fig. 7A). In contrast, the proportion of patients with hypertension was independent on CKD stage (Fig. 7B) confirming our above findings. Glomerular (Fig. 7C) and tubular C3c (Fig. 7D) were significantly lower in CKD stages 1 and 2 than higher CKD stages, whereas no such association was observed for $\mathrm{Clq}$ (Fig. 7E, F).

(C, D) compartment. Similarly, C1q deposition was also analyzed in biopsies from patients with $\operatorname{IgAN}(\mathbf{E}, \mathbf{G})$ and all investigated biopsies with kidney diseases $(\mathbf{F}, \mathbf{H})$ in the glomerular $(\mathbf{E}, \mathbf{F})$ and tubulointerstitial $(\mathbf{G}, \mathbf{H})$ compartment. Significant differences are marked by asterisks: ${ }^{*} p<0.05 ;{ }^{* *} p<0.01$ and ${ }^{* * *} p<0.001$ indicate significant differences. BP, blood pressure.

(J) and glomerular C3c with serum creatinine (K) and to a weaker extent glomerular $\mathrm{C} 3 \mathrm{c}$ with proteinuria $(\mathbf{L})$. Significant differences are marked by asterisks: ${ }^{* *} p<0.01$ and ${ }^{* * *} p<0.001$ indicate significant differences between patients with normal BP and high BP or significant correlations. BP, blood pressure; GSI, glomerulosclerosis; TSI, tubulointerstitial injury; VSI, vascular injury; IF/TA, interstitial fibrosis/tubular atrophy.

(For figure see next page.) 


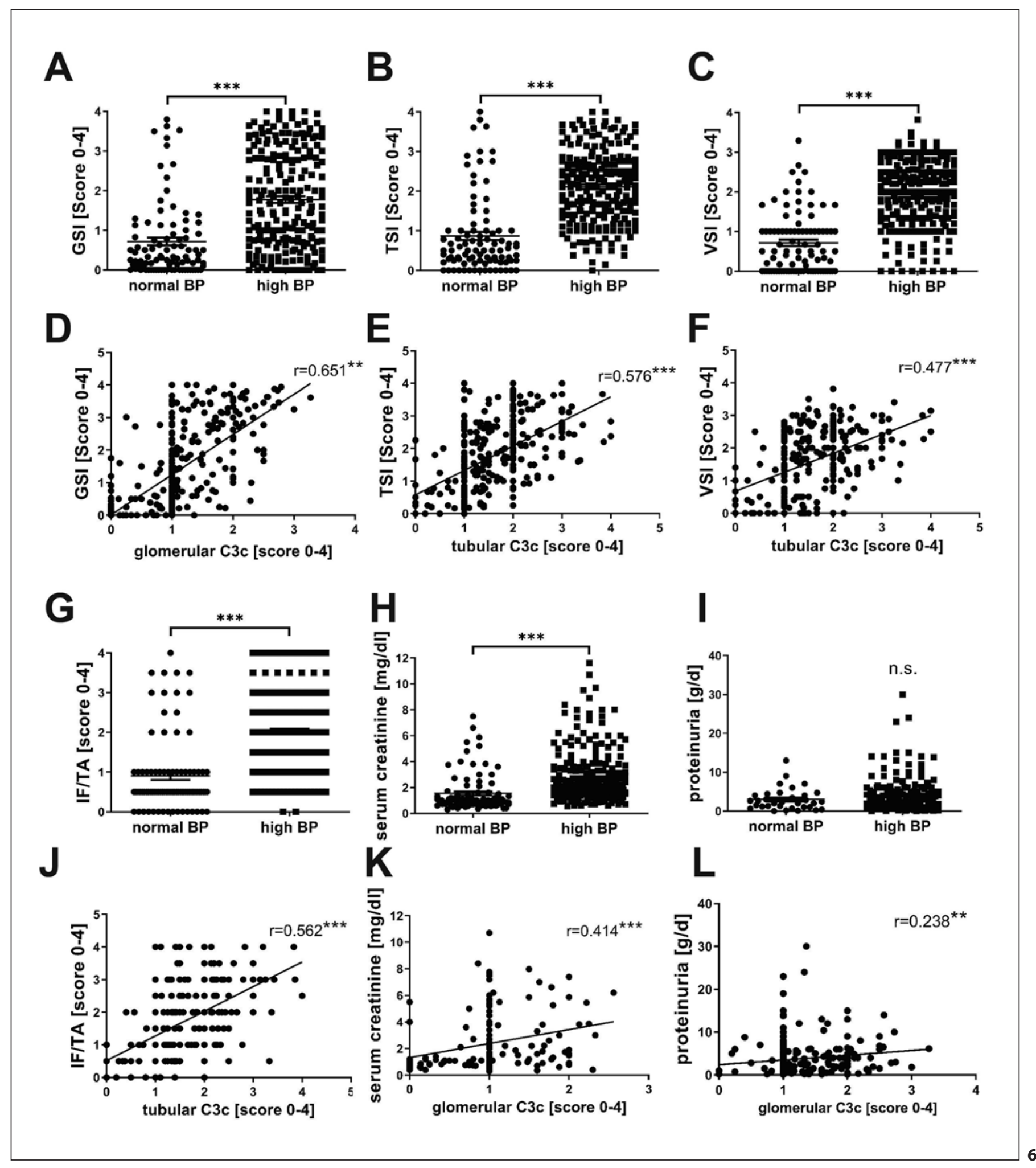


Fig. 7. Renal C3 deposition in human biopsies, but not hypertension is correlated with CKD stage. Mean age of patients with CKD increased with increasing CKD stage (A), while the percentage of hypertensive patients was not related to CKD stage (B). $\mathrm{C} 3 \mathrm{c}(\mathbf{C}, \mathbf{D})$ but not C1q (E, F) nicely correlated with different CKD stages. Glomerular C3c (C), tubular C3c (D), glomerular $\mathrm{C} 1 \mathrm{q}(\mathbf{E})$, and tubular C1q (F). Significant differences are marked by asterisks: ${ }^{*} p<$ 0.05 ; ${ }^{*} p<0.01$; and ${ }^{* * *} p<0.001$ indicate significant differences between groups connected by brackets.
A

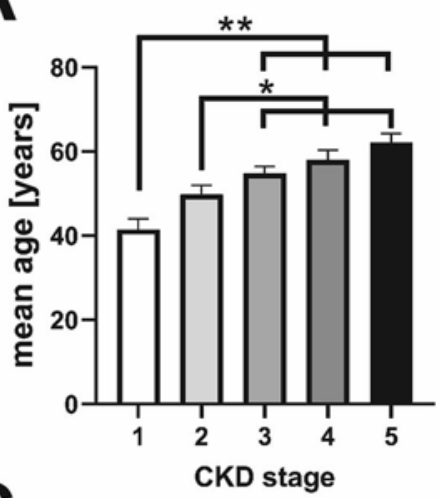

C

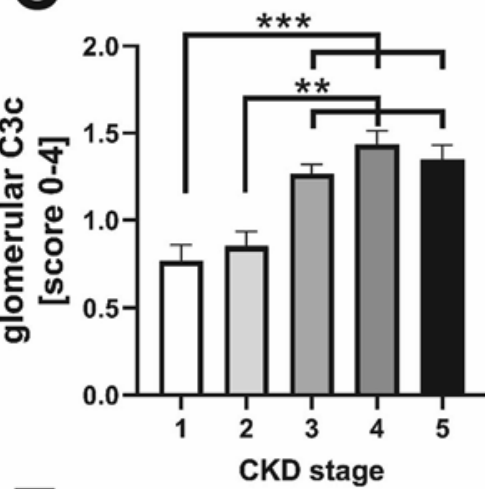

E

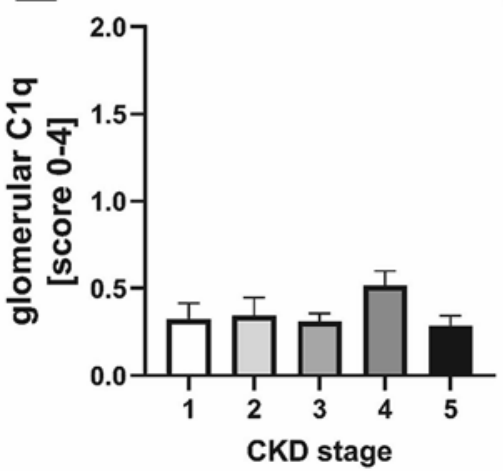

B

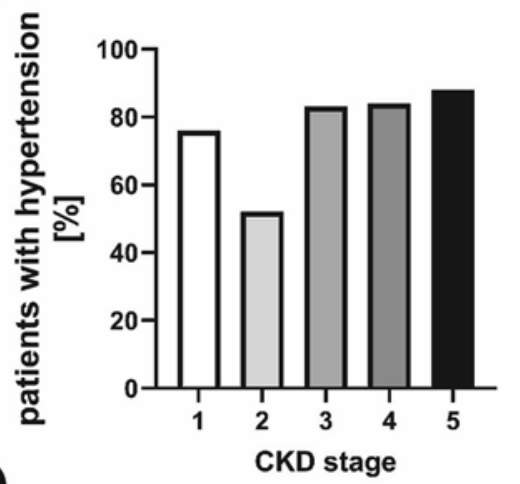

D

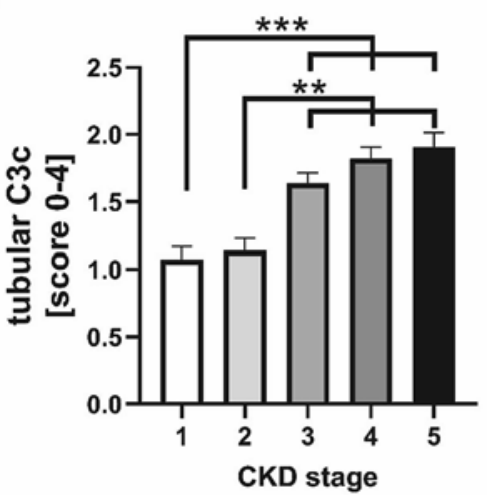

$\mathbf{F}$

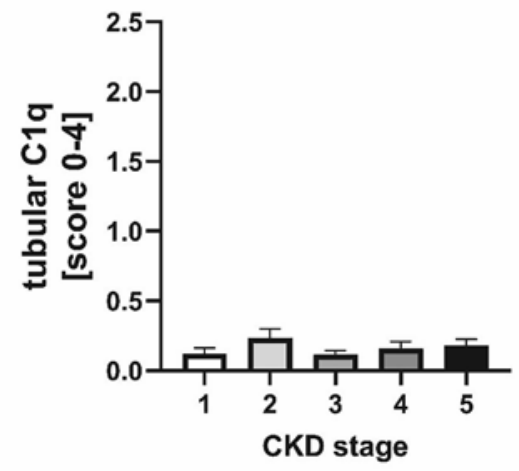

\section{Discussion}

Progression of renal disease, especially tubulointerstitial damage, was shown to be mediated, at least in part, by complement activation [28], but the role of complement deposition in hypertension and renal damage is still unclear. In our study, we found increased renal deposition of complement factors, particularly of C3c, which strongly correlated with increased kidney injury and inferior renal function. In addition, very frequently kidney disease is associated with the occurrence of hypertension which itself is linked to increased risk of cardiovascular disease and progression of CKD [9]. Here, we showed higher renal deposition of complement cleavage products $\mathrm{C} 1 \mathrm{q}$, as a marker of the classical activation pathway, and $\mathrm{C} 3 \mathrm{c}$, a component of all 3 activation pathways, in kidneys from hypertensive patients with different renal diseases than normotensive pa- 
tients. There is some evidence that complement plays an important role in the development and worsening of hypertension, but the role of renal complement deposition in this process is unknown. In human renal biopsies and our hypertensive overload rat model, it has become apparent that $\mathrm{C} 3 \mathrm{c}$ is more frequently detectable than $\mathrm{Clq}$ suggesting that the classical pathway is not exclusively responsible for complement activation in renal disease. Nonetheless, C1q was found to be critically involved in hypertensive arterial remodeling via activation of $\beta$-catenin signaling [29]. The question if complement induces hypertension or hypertension activates complement remains to date unclear. In this study, we observed a strong correlation of renal complement deposition, particularly of factor $\mathrm{C} 3 \mathrm{c}$, with the degree of renal injury and function. Most likely hypertension leads to further activation of the complement system, but there are some reports supporting the hypothesis that complement is not only secondary to but also causative for hypertension. In a longitudinal study with a follow-up of 15 years, the incidence of hypertension was significantly higher in patients with elevated plasma complement C3 levels at baseline suggesting a role for plasma C3 in the pathogenesis of hypertension [10]. Additionally, patients with therapy-resistant arterial hypertension had significantly higher C3 plasma levels compared to patients with controlled hypertension [11]. In contrast, in a population-based cohort study elevated plasma C3 was associated with increased incidence of first hospitalization due to CKD but independent of BP [12]. However, the latter study was restricted to the investigation of C3 plasma levels, whilst local expression of complement factors and complement activation were not investigated. C3 cleavage products, that is, $\mathrm{C} 3 \mathrm{c}$, were not only passively deposited but C3 mRNA was also locally expressed in diseased kidneys from hypertensive patients and SNX rats. We did not determine the source of renal $\mathrm{C} 3$, however, but there are reports that beside the liver the majority of $\mathrm{C} 3$ mRNA is expressed in monocytes and macrophages [30]. In addition fibroblasts, epithelial and endothelial cells have also been reported to be potential extrahepatic C3-sources [31]. Complement activation was also seen in an angiotensin II-induced hypertension model in mice. In spontaneously hypertensive rats, C3 deficiency could abolish salt-sensitive hypertension [18], indicating that $\mathrm{C} 3$ is critical involved in hypertensioninducing pathways. However, these effects can also be mediated further downstream in the complement cascade. Using C5a receptor (C5aR) deficient mice Zhang et al. [32] demonstrated that C5aR-signaling plays a pathological role in cardiac inflammation and remodeling. Although $\mathrm{C} 5 \mathrm{aR}$ inhibition did not affect the elevated BP induced by angiotensin II, cardiac damage, as assessed by cardiac hypertrophy, inflammation, and perivascular fibrosis could be reduced [33]. In our SNX rat model of hypertension, we confirmed that renal complement deposition correlated well with renal damage but also with cardiac hypertrophy and BP. Complement-induced hypertension was mediated via complement receptors for C3a and C5a. Regulatory Tregs can inhibit activation of effector Tregs and exerts anti-inflammatory effects. In the angiotensin II-induced model of hypertension, Tregs have been shown to be an important modulator of BP and prevention of end-organ damage $[15,34]$. Angiotensin II-treatment stimulated expression of $\mathrm{C} 3 \mathrm{a}$ and $\mathrm{C} 5 \mathrm{a}$ receptor on Tregs leading to a reduced Tregs number in wild-type mice, but not in $\mathrm{C} 3 \mathrm{a}$ and C5a receptor double knockout mice [35]. In addition, these double knockout mice developed blunted hypertension and less renal fibrosis and glomerular injury [35]. An association of complement activation and glomerular injury and renal fibrosis, as assessed by glomerulosclerosis and IF/TA, was also confirmed in our biopsy study. Mechanistically, BP increase was induced by C3-mediated activation of the renin-angiotensin system in vascular smooth muscle cells with downstream molecules such as TGF-B/PDGF-A and by changing the phenotype of mesenchymal cells and induction of epithelial-to-mesenchymal transition [31]. Complement signaling was also targeted in other hypertensive diseases. In normal pregnancy, complement activation is present but is excessive in hypertensive disorders of pregnancy and genetic polymorphisms of complement proteins seem to predispose to preeclampsia [36]. Inhibition of complement signaling by treatment with soluble complement receptor 1 resulted in attenuated hypertension in a placental ischemia model of preeclampsia [37].

\section{Limitations of the Study}

Although we tried to strengthen our findings in human renal biopsies of various renal diseases with and without arterial hypertension by the incorporation of an appropriate animal model to prove an association between complement deposition in kidney tissue and hypertension, we have to acknowledge several limitations and selection biases. In the human study, we face the limitation of a variable cohort with numerous different confounders that cannot be clearly identified or 
excluded. In order to address this aspect and to harmonize the study group, we also analyzed the correlation of complement with renal fibrosis (IF/TA) as a surrogate marker of nephron loss and progression of the underlying renal disease as well as with CKD stages where we could show similar findings. Moreover, data on duration of the respective diseases or current medical treatment were not systematically available leaving these aspects as another potential confounder that we have to mention. Since we formally excluded those few patients without clinically well-documented BP, it is likely that those patients might have either no hypertension or controlled hypertension to the point that they might come to regular follow-up which might potentially cause selection bias. In contrast to the animal experiment in our human cohort proteinuria at the time of renal biopsy was not consistently reported for all patients so that we could only include $2 / 3$ of all patients for the respective analyses which may affect some of the results. Of note, proteinuria was not significantly higher in renal patients with and without high BP making a significant bias of proteinuria unlikely.

\section{Conclusion}

Taken together, our study underlines a strong association of complement deposition with hypertension but also renal damage in human and experimental renal disease. Although we could not answer the question whether complement deposition is causative of the development of hypertension, the data support an involvement of complement activation in the pathogenesis or progression of hypertension in various kidney diseases, implying a potential role for complement-inhibiting strategies for future management of therapy-refractory cases of hypertension and renal disease. However, additional studies are needed to address the exact role of complement in pathogenesis of hypertensive diseases.

\section{Acknowledgements}

The present work was performed in (partial) fulfillment of the requirements for obtaining the degree "Dr. med. dent." from the FAU Erlangen-Nürnberg for L.-M. Fischer. The technical assistance of S. Söllner, K. Schmitt, A. Kosel, M. Klewer, C. Grigo, and M. Reutelshöfer is gratefully acknowledged.

\section{Statement of Ethics}

The study on archived human renal biopsies was conducted according to the principles of the Declaration of Helsinki and approved by the local Ethics Committee of the FAU Erlangen-Nürnberg (reference number 4415). The experimental protocol for the animal studies was approved by the German regional committee for animal care and use, which is equivalent to the US IACUC, and authorized by the governmental department ("Regierung von Mittelfranken" Permit number: 54-2532.1-18/11) prior the animal studies were performed in strict accordance with the German welfare act.

\section{Conflict of Interest Statement}

The authors have no conflicts of interest to declare.

\section{Funding Sources}

This study was funded by the Deutsche Forschungsgemeinschaft (German Research Foundation), project number 387509280 , SFB 1350 TP C2, and supported by a grant from the "Deutsche Hochdruckliga" to K. Benz.

\section{Author Contributions}

L.-M.F. conducted all experimental work on human biopsies, analyzed data, and wrote the manuscript; L.A.R. carried out immunohistological staining and data analysis for rat experiments; F.F. performed the statistical analysis; M.B.H. and K.A. made the diagnoses, performed some of the analyses, and wrote the paper; K.B. and C.D. conceived and designed the study, analyzed data, and wrote the manuscript. All the authors had proofread the manuscript.

\section{References}

1 Couser WG. Basic and translational concepts of immune-mediated glomerular diseases. J Am Soc Nephrol. 2012 Mar;23(3):381-99.

2 Kojouharova M, Reid K, Gadjeva M. New insights into the molecular mechanisms of classical complement activation. Mol Immunol. 2010 Aug;47(13):2154-60.

3 Dobo J, Pal G, Cervenak L, Gal P. The emerging roles of mannose-binding lectin-associated serine proteases (MASPs) in the lectin pathway of complement and beyond. Immunol Rev. 2016 Nov;274(1):98-111.

4 Pangburn MK, Schreiber RD, Muller-Eberhard HJ. Formation of the initial C3 convertase of the alternative complement pathway. Acquisition of C3b-like activities by spontaneous hydrolysis of the putative thioester in native C3. J Exp Med. 1981 Sep 1;154(3):856-67.

5 Ricklin D, Hajishengallis G, Yang K, Lambris JD. Complement: a key system for immune surveillance and homeostasis. Nat Immunol. 2010 Sep;11(9):785-97.

6 Nozal P, Lopez-Trascasa M. Autoantibodies against alternative complement pathway proteins in renal pathologies. Nefrologia. 2016 Sep-Oct;36(5):489-95.

7 Lau KK, Suzuki H, Novak J, Wyatt RJ. Pathogenesis of Henoch-Schönlein purpura nephritis. Pediatr Nephrol. 2010 Jan;25(1):1926. 
8 Wenzel U, Turner JE, Krebs C, Kurts C, Harrison DG, Ehmke H. Immune mechanisms in arterial hypertension. J Am Soc Nephrol. 2016 Mar;27(3):677-86.

9 Drawz PE, Beddhu S, Kramer HJ, Rakotz M, Rocco MV, Whelton PK. Blood pressure measurement: a KDOQI perspective. Am J Kidney Dis. 2020 Mar;75(3):426-34.

10 Engstrom G, Hedblad B, Berglund G, Janzon L, Lindgarde F. Plasma levels of complement C3 is associated with development of hypertension: a longitudinal cohort study. J Hum Hypertens. 2007 Apr;21(4):276-82.

11 Magen E, Mishal J, Paskin J, Glick Z, Yosefy C, Kidon M, et al. Resistant arterial hypertension is associated with higher blood levels of complement $\mathrm{C} 3$ and $\mathrm{C}$-reactive protein. J Clin Hypertens. 2008 Sep;10(9):677-83.

12 Bao X, Borne Y, Muhammad IF, Schulz CA, Persson M, Orho-Melander M, et al. Complement $\mathrm{C} 3$ and incident hospitalization due to chronic kidney disease: a population-based cohort study. BMC Nephrol. 2019 Feb 21; 20(1):61.

13 Klanke B, Cordasic N, Hartner A, Schmieder RE, Veelken R, Hilgers KF. Blood pressure versus direct mineralocorticoid effects on kidney inflammation and fibrosis in DOCAsalt hypertension. Nephrol Dial Transplant. 2008 Nov;23(11):3456-63.

14 Chen L, Fukuda N, Shimizu S, Kobayashi H, Tanaka S, Nakamura Y, et al. Role of complement 3 in renin generation during the differentiation of mesenchymal stem cells to smooth muscle cells. Am J Physiol Cell Physiol. 2020 May 1;318(5):C981-C990.

15 Kvakan H, Kleinewietfeld M, Qadri F, Park JK, Fischer R, Schwarz I, et al. Regulatory T cells ameliorate angiotensin II-induced cardiac damage. Circulation. 2009 Jun 9;119(22): 2904-12.

16 Ruan CC, Ge Q, Li Y, Li XD, Chen DR, Ji KD, et al. Complement-mediated macrophage polarization in perivascular adipose tissue contributes to vascular injury in deoxycorticosterone acetate-salt mice. Arterioscler Thromb Vasc Biol. 2015 Mar;35(3):598-606.
17 Carney EF. Hypertension: role of $\mathrm{C} 3 \mathrm{aR}$ and C5aR in treg cells. Nat Rev Nephrol. 2018 Apr; 14(4):214

18 Negishi E, Fukuda N, Otsuki T, Katakawa M, Komatsu K, Chen L, et al. Involvement of complement 3 in the salt-sensitive hypertension by activation of renal renin-angiotensin system in spontaneously hypertensive rats. Am J Physiol Renal Physiol. 2018 Dec 1; 315(6):F1747-F1758

19 Dimmler A, Haas CS, Cho S, Hattler M, Forster C, Peters H, et al. Laser capture microdissection and real-time PCR for analysis of glomerular endothelin-1 gene expression in mesangiolysis of rat anti-Thy 1.1 and murine Habu Snake Venom glomerulonephritis. Diagn Mol Pathol. 2003 Jun;12(2):108-17.

20 Diez M, Abdelmagid N, Harnesk K, Strom M, Lidman O, Swanberg M, et al. Identification of gene regions regulating inflammatory $\mathrm{mi}-$ croglial response in the rat CNS after nerve injury. J Neuroimmunol. 2009 Jul 25;212(12):82-92.

21 Hainz N, Thomas S, Neubert K, Meister S, Benz K, Rauh M, et al. The proteasome inhibitor bortezomib prevents lupus nephritis in the NZB/W F1 mouse model by preservation of glomerular and tubulointerstitial architecture. Nephron Exp Nephrol. 2012; 120(2):e47-58.

22 Solez K, Racusen LC. The Banff classification revisited. Kidney Int. 2013 Feb;83(2):201-6.

23 KDIGO clinical practice guideline for the management of blood pressure in chronic kidney disease. Kidney Int Suppl. 2012 Dec; 2(5):343.

24 Levey AS, Stevens LA, Schmid CH, Zhang YL, Castro AF 3rd, Feldman HI, et al. A new equation to estimate glomerular filtration rate. Ann Intern Med. 2009 May 5;150(9):604-12.

25 Group KCW. KDIGO 2012 clinical practice guideline for the evaluation and management of chronic kidney disease. Kidney Int Suppl. 2013;3:1-150.

$26 \mathrm{Ju} \mathrm{W}$, Greene CS, Eichinger F, Nair V, Hodgin JB, Bitzer M, et al. Defining cell-type specificity at the transcriptional level in human disease. Genome Res. 2013 Nov;23(11):1862-73.

27 Barratt J, Feehally J, Smith AC. Pathogenesis of IgA nephropathy. Semin Nephrol. 2004 May;24(3):197-217.
28 Hsu SI, Couser WG. Chronic progression of tubulointerstitial damage in proteinuric renal disease is mediated by complement activation: a therapeutic role for complement inhibitors? J Am Soc Nephrol. 2003 Jul;14(7 Suppl 2):S186-91.

29 Sumida T, Naito AT, Nomura S, Nakagawa A, Higo T, Hashimoto A, et al. Complement C1q-induced activation of $\beta$-catenin signalling causes hypertensive arterial remodelling. Nat Commun. 2015;6:6241.

30 de Bruijn MH, Fey GH. Human complement component C3: cDNA coding sequence and derived primary structure. Proc Natl Acad Sci U S A. 1985 Feb;82(3):708-12.

31 Chen L, Fukuda N, Matsumoto T, Abe M. Role of complement 3 in the pathogenesis of hypertension. Hypertens Res. 2020 Apr; 43(4):255-62.

32 Zhang C, Li Y, Wang C, Wu Y, Cui W, Miwa $\mathrm{T}$, et al. Complement 5 a receptor mediates angiotensin II-induced cardiac inflammation and remodeling. Arterioscler Thromb Vasc Biol. 2014 Jun;34(6):1240-8.

33 Zhang C, Li Y, Wang C, Wu Y, Du J. Antagonist of C5aR prevents cardiac remodeling in angiotensin II-induced hypertension. Am J Hypertens. 2014 Jun;27(6):857-64.

34 Barhoumi T, Kasal DA, Li MW, Shbat L, Laurant $\mathrm{P}, \mathrm{Neves} \mathrm{MF}$, et al. T regulatory lymphocytes prevent angiotensin II-induced hypertension and vascular injury. Hypertension. 2011 Mar;57(3):469-76.

35 Chen XH, Ruan CC, Ge Q, Ma Y, Xu JZ, Zhang ZB, et al. Deficiency of Complement $\mathrm{C} 3 \mathrm{a}$ and $\mathrm{C} 5 \mathrm{a}$ receptors prevents angiotensin II-induced hypertension via regulatory $\mathrm{T}$ cells. Circ Res. 2018 Mar 30;122(7):970-83.

36 Regal JF, Burwick RM, Fleming SD. The complement system and preeclampsia. Curr Hypertens Rep. 2017 Oct 18;19(11):87.

37 Regal JF, Lund JM, Wing CR, Root KM, McCutcheon L, Bemis LT, et al. Interactions between the complement and endothelin systems in normal pregnancy and following placental ischemia. Mol Immunol. 2019 Oct;114: $10-8$. 